Check for updates

Cite this: Phys. Chem. Chem. Phys., 2018, 20, 4422

Received 22nd November 2017,

Accepted 9th January 2018

DOI: $10.1039 / c 7 c p 07871 d$

rsc.li/pccp

\section{Graphene induced electrical percolation enables more efficient charge transport at a hybrid organic semiconductor/graphene interface $\dagger$}

\author{
Nicolas Boulanger, (D) ${ }^{a}$ Victor Yu, ${ }^{b}$ Michael Hilke, ${ }^{b}$ Michael F. Toney (iD c and \\ David R. Barbero iD *a
}

\begin{abstract}
Self-assembly of semiconducting polymer chains during crystallization from a liquid or melt dictates to a large degree the electronic properties of the resulting solid film. However, it is still unclear how charge transport pathways are created during crystallization. Here, we performed complementary in situ electrical measurements and synchrotron grazing incidence X-ray diffraction (GIXD), during slow cooling from the melt of highly regio-regular poly(3-hexylthiophene) (P3HT) films deposited on both graphene and on silicon. Two different charge transport mechanisms were identified, and were correlated to the difference in crystallites' orientations and overall amount of crystallites in the films on each surface as molecular self-assembly proceeded. On silicon, a weak charge transport was enabled as soon as the first edge-on lamellae formed, and further increased with the higher amount of crystallites (predominantly edge-on and randomly oriented lamellae) during cooling. On graphene however, the current remained low until a minimum amount of crystallites was reached, at which point interconnection of conducting units (face-on, randomly oriented lamellae and tie-chains) formed percolated conducting pathways across the film. This lead to a sudden rapid increase in current by $\approx 10$ fold, and strongly enhanced charge transport, despite a much lower amount of crystallites than on silicon.
\end{abstract}

\section{Introduction}

Graphene is an outstanding material formed of a 2 dimensional layer of carbon atoms, in which the delocalization of $\pi$ electrons, and its ultrafast charge transport, opens up the possibility to transfer charges to and from a conjugated polymer layer for organic hybrid opto-electronic applications. ${ }^{1-5}$ Combining graphene with the semiconducting polymer P3HT into OFETs, OPVs and other electronic devices has been recently demonstrated. ${ }^{3,5-8}$ However, the performance of such hybrid devices relies on the ability of the graphene/semiconducting polymer composite layer to transport charges efficiently. Self-assembly and molecular packing strongly influence the electronic properties of the organic film, and therefore control of the overall crystallinity but also of the crystallites' orientation, size and interplanar spacing, is critical.

It is often believed that highly crystalline films are necessary in order to enhance charge transport in organic semiconductors, such as P3HT. Although a large amount of crystallites has been

\footnotetext{
${ }^{a}$ Nano-Engineered Materials \& Organic Electronics Laboratory, Umeå University, Umeå, Sweden. E-mail: david.barbero@umu.se

${ }^{b}$ Department of Physics, McGill University, Montréal, Québec, H3A 2T8, Canada

${ }^{c}$ Stanford Synchrotron Radiation Lightsource, Menlo Park, CA, USA

$\dagger$ Electronic supplementary information (ESI) available: Experimental procedures for characterization of the samples. See DOI: $10.1039 / \mathrm{c} 7 \mathrm{cp} 07871 \mathrm{~d}$
}

shown to help with the interconnection of conducting crystalline domains (e.g. by tie-chains), it is also important to distinguish between the different types of crystallite orientations (e.g. edge-on vs. face-on) which help direct charges in a specific direction. A less crystalline film can be more conductive due to better interconnection of preferentially oriented crystallites. ${ }^{9}$ In the edge-on orientation, the backbone and side-chains of the polymer are stacked perpendicularly to the surface of the substrate, with the $\pi-\pi$ stacking parallel to the substrate, as shown in Fig. 1a. In the face-on orientation, the polymer backbone and side-chains are parallel to the substrate surface, with the $\pi-\pi$ stacking pointing in the vertical direction, as shown in Fig. 1b. It has been shown that P3HT tends to form in-plane $\pi-\pi$ stacking (edge-on lamellae) on weakly interacting surfaces (e.g. silicon oxide), which is useful for devices where charges must be transported in the plane of the film (e.g. OFETs). ${ }^{10-13}$ However, in order to produce efficient out-of-plane charge transport in a diode configuration (OLEDs or OPVs), crystallites should be oriented either with their chain backbones, or with their $\pi$ stacking in the out-of-plane direction. ${ }^{11,14,15}$

Graphene has been shown to favor vertical $\pi$ stacking in a polythiophene semiconductor due to the strong $\pi-\pi$ interactions between the two materials. ${ }^{16}$ Graphene was previously used to enhance the efficiency of a hybrid graphene/organic photovoltaic 

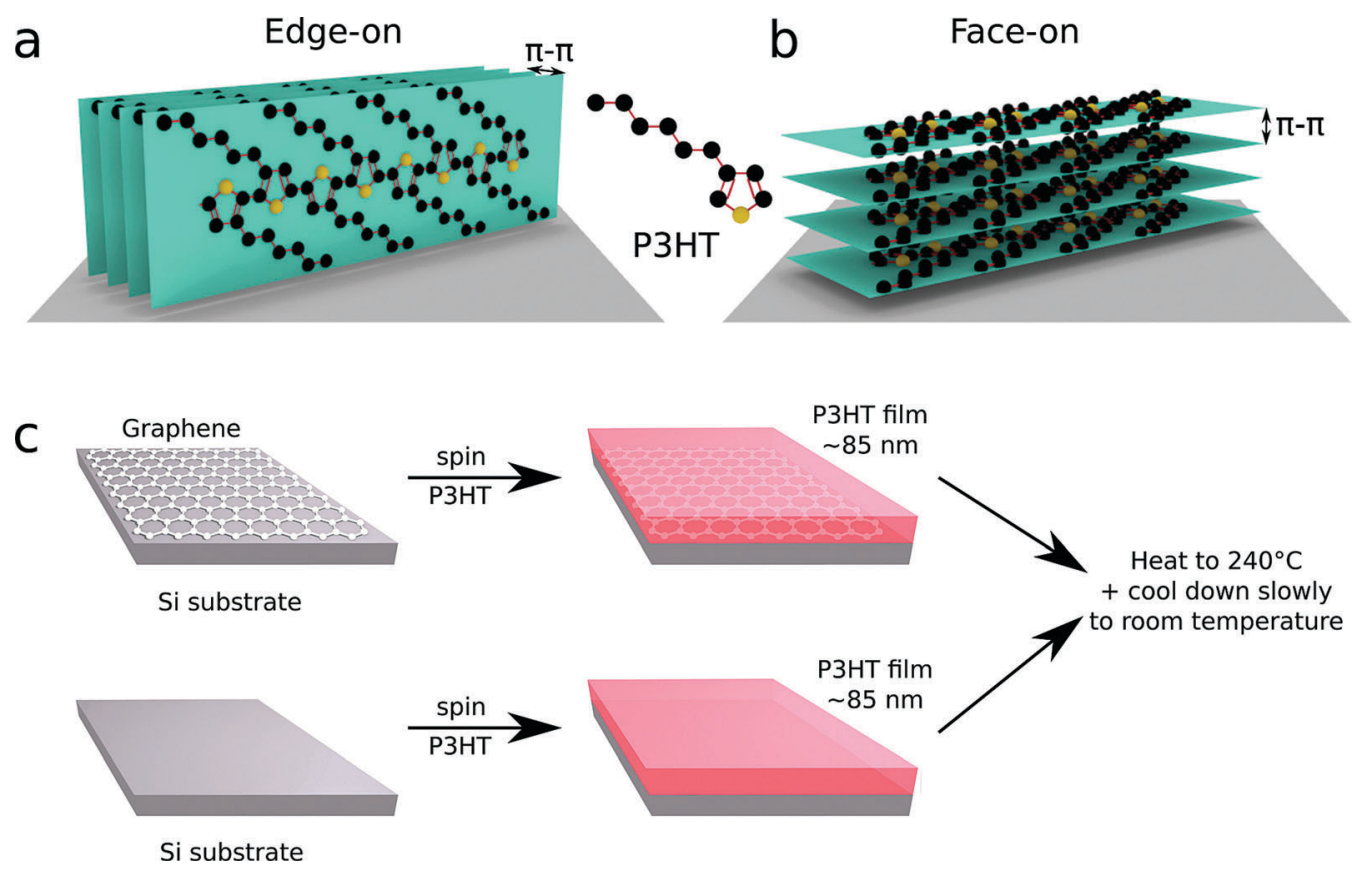

Fig. 1 P3HT molecule and orientations as well as preparation and annealing of the graphene/P3HT and silicon/P3HT samples. (a) Edge-on orientation with an in-plane $\pi-\pi$ stacking, and (b) face-on orientation with a vertical $\pi-\pi$ stacking. A P3HT molecule with the black carbon atoms and the yellow sulfur atom is represented in (a). (c) Thin films of P3HT were spun from solution onto both silicon and graphene surfaces, heated up to $240{ }^{\circ} \mathrm{C}$ for 5 min and slowly cooled down to room temperature.

device, where the graphene sheet helped the epitaxial growth of pentacene crystals, resulting in a longer exciton diffusion length and an improved charge transfer between the polymer and the graphene substrate. ${ }^{17} \mathrm{P} 3 \mathrm{HT}$ has also been grafted to a graphene sheet using an esterification reaction, and the resulting compound has been used in combination with $\mathrm{C}_{60}$ to create bilayer photovoltaic devices. ${ }^{18}$ This resulted in a $200 \%$ increase in efficiency compared to a device without graphene, due to an enhanced charge transfer between the P3HT and the graphene, leading to improved charge extraction.

In order to produce fast charge transport in graphene/P3HT hybrid devices, one needs to understand which critical parameters influence charge transport and the orientation of the crystallites formed in the semiconducting polymer film during crystallization. Another important factor for efficient charge transport is the good energetic alignment between the graphene, silicon and P3HT interfaces with the Fermi level of graphene lying in between the work functions of silicon $(\approx-5.1 \mathrm{eV})$ and P3HT ( $\approx-4.7$ to $-5.1 \mathrm{eV})$. This enables efficient hole injection from both silicon and graphene into the semiconducting polymer layer. ${ }^{19}$ Knowledge of how crystallization and charge transport are interlinked during cooling is therefore important to understand the mechanism of charge transport of a semiconducting polymer film deposited on different interacting surfaces (e.g. silicon $v s$. graphene).

Here we show the relationship between crystallization of P3HT on both graphene and silicon, and charge transport in the polymer film, by performing both GIXD and electrical measurement in situ as a function of temperature during cooling from a disordered (non-crystalline) state. Two different charge transport mechanisms were identified, and were correlated to the difference in crystallites' orientations and overall amount of crystallites in the films on each surface as a function of the cooling temperature. Surprisingly, on graphene the amount of crystallites and the current measured remained lower than on silicon, until a sudden rapid increase in current by $\approx 10$ fold occurred around $60^{\circ} \mathrm{C}$, corresponding to a minimum value of the $\pi-\pi$ spacing, and a minimum amount of crystallites necessary for percolation to occur. Once the percolated pathways were formed, a strong enhancement in charge transport was measured on graphene, despite the lower amount of crystallites, resulting in enhanced charge carrier mobility.

\section{Experimental section}

\section{Materials and sample preparation}

Poly-3-hexylthiophene (P3HT) powder (98\% RR, $\left.M_{\mathrm{w}}=32 \mathrm{kD}\right)$ was purchased from American Dye Source Inc. Graphene single layers were synthesized by chemical vapor deposition (CVD), and transferred onto a silicon substrate by etching the copper and floating the graphene in DI water. Details of the synthesis and transfer procedure of graphene can be found elsewhere. ${ }^{19-21}$ P3HT films $85 \mathrm{~nm}$ thick were spun dry from a dilute solution of dichlorobenzene (o-DCB) onto either graphene sheets deposited on silicon, or onto silicon substrates directly. The films were annealed in inert helium atmosphere to $240{ }^{\circ} \mathrm{C}$ for $5-10 \mathrm{~min}$, followed by slow cooling $\left(\approx 2-3{ }^{\circ} \mathrm{C} \mathrm{min}^{-1}\right)$ to room temperature (see Fig. 1c), while they were irradiated by an X-ray beam at given temperatures on the synchrotron beamline (see below). 


\section{Synchrotron X-ray diffraction}

The crystalline orientations in the P3HT films was measured in situ during slow cooling by $2 \mathrm{D}$ grazing-incidence $\mathrm{X}$-ray diffraction (see in ESI, $\uparrow$ Fig. S1a), at the Stanford Synchrotron Radiation Lightsource (SSRL) (Menlo Park, CA, USA) on beamline 11-3. The beam energy was $12.7 \mathrm{keV}$, and the incident angle $\alpha$ was $0.13^{\circ}$, which enabled penetration of the X-ray beam inside the whole thickness of the P3HT films. In order to limit sample degradation, measurements were carried out in an inert atmosphere (helium) for $20 \mathrm{~s}$ per exposure. Measurements were also done on the 2-1 beamline in the Bragg configuration, in order to detect crystallites in the out-of-plane orientation. They were carried out using a point detector, with a $2 \mathrm{~s}$ exposure per measured datapoint. Measurements showed no noticeable degradation of the samples. Grazing incidence X-ray diffraction confirmed that the films were completely disordered at $240{ }^{\circ} \mathrm{C}$, and showed no diffraction pattern (Fig. S1b and c, ESI $\dagger$ ). The diffracted intensities were normalized by exposure time, beam energy, and volume probed in order to allow a direct comparison between each film. The background was also subtracted from each data set, and the peaks were fitted with either a PseudoVoigt or a Lorentzian fit. ${ }^{22}$ Because of the weak diffraction from the films at temperatures above $210{ }^{\circ} \mathrm{C}$, the X-ray diffraction data is only shown for temperatures below $210{ }^{\circ} \mathrm{C}$. Compared to a traditional X-ray diffraction set-up, the use of a synchrotron beam is advantageous for this type of measurements for several reasons. The first advantage is the specific set-up of BL 11-3 which is fine tuned for measuring thin polymer films, including a dynamic and sensitive large area detector, an inert chamber to limit sample damage, and the possibility to heat and cool the sample sensitively to high enough temperatures. Second, the sample-to-detector distance can be adjusted from 80 to $550 \mathrm{~mm}$, which is not always possible to do on a traditional "in-house" set-up. Third, the high intensity of the synchrotron beam is ideally suited to the measurement of weakly diffracting polymer thin-films. It indeed enabled much shorter exposure times which was important to limit sample damage, and also to reduce measurement time since these GIXD measurements typically lasted 6-8 hours per sample due to the slow cooling rate. Increasing the exposure time from $20 \mathrm{~s}$ to 10-100 min per temperature point on a traditional set-up not only creates a substantial increase in measurement time, but it can also potentially produce damage to the sample (especially at elevated temperatures).

\section{Electrical characterization}

Electrical characterization was also performed in situ in a parallel set of experiments by measuring the current flowing vertically across the film's thickness at a low voltage bias of $1 \mathrm{~V}$ during cooling (Fig. 2a) using a Keithley 2450 sourcemeter. The resulting current between the conducting substrate and the electrode at the top of the film was measured every 5 to 10 degrees during slow cooling down of the films. A smooth and flexible polydimethylsiloxane (PDMS) slab, onto which $\approx 200 \mathrm{~nm}$ gold film was slowly evaporated in vacuum, was used as the top electrode. Metal coated PDMS electrodes enable very good conformal contact with the top surface of the film, and this method has been previously established as an alternative to direct metal evaporation. ${ }^{23}$ Gold was chosen because its work function is reported to be $\approx-5.1 \mathrm{eV},{ }^{24}$ which is very close to the HOMO of P3HT $(\approx-4.7$ to $-5.1 \mathrm{eV}),{ }^{25,26}$ thereby providing a very low energetic barrier for hole transport with P3HT. The charge-carrier mobility $(\mu)$ of the P3HT films were extracted from current density-voltage $(J-U)$ characteristics at room temperature in the vertical direction (diode configuration) at saturation (3.5 V) using the classical Mott-Gurney $(\mathrm{M}-\mathrm{G})$ equation (eqn (1)): ${ }^{27}$

$$
\text { Mott-Gurney: } \mu=\frac{8 J L^{3}}{9 \varepsilon U^{2}}
$$

where $\mu$ is the charge-carrier mobility, $J$ the current density, $U$ the applied voltage, $L$ the sample thickness, and $\varepsilon$ the dielectric constant of the film. This equation is used to describe space-charge-limited current (SCLC) measured in the drift regime in organic semiconductors, in which the SCLC current varies quadratically with the voltage (as shown in ESI, $\dagger$ Fig. S5). Note that due to the difficulty to perform electrical measurements and obtaining meaningful currents on disordered films (at higher temperatures, $>180{ }^{\circ} \mathrm{C}$ ), the values of current shown here are for temperatures below $180{ }^{\circ} \mathrm{C}$.

\section{Results and discussion}

During cooling of the film on silicon, the current slowly and continuously increased from a temperature of $\approx 210{ }^{\circ} \mathrm{C}$ to $\approx 100{ }^{\circ} \mathrm{C}$
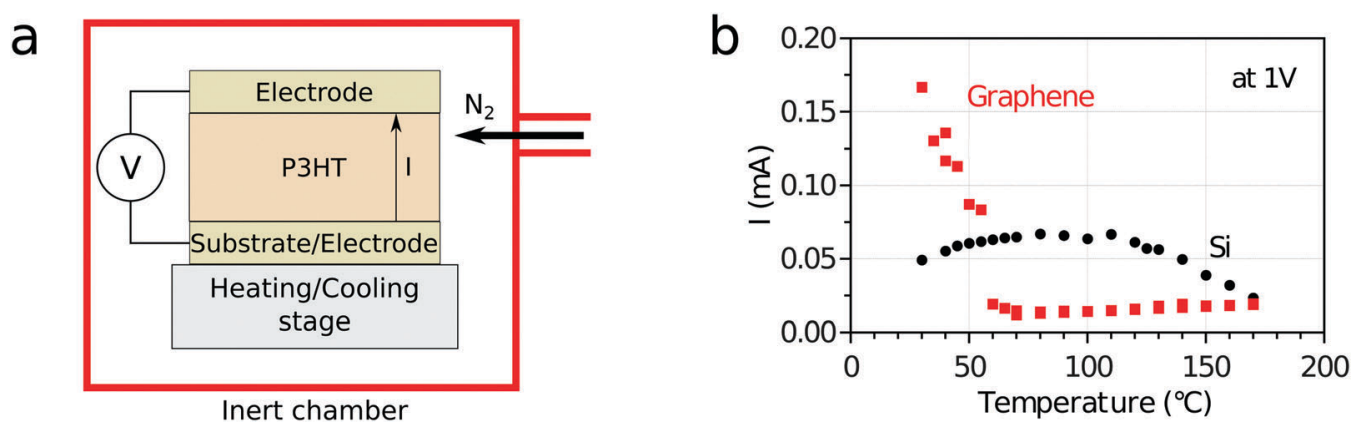

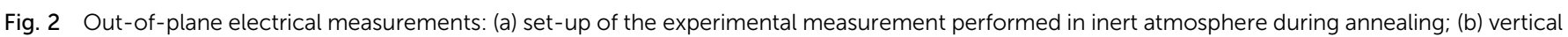
current vs. temperature measured during cooling from the melt on both graphene and silicon at $1 \mathrm{~V}$ applied bias. 
at which point it reached a maximum value (Fig. 2b). Upon further cooling, the current stabilized from $\approx 100{ }^{\circ} \mathrm{C}$ to $\approx 80{ }^{\circ} \mathrm{C}$ and then slightly dropped at room temperature. A very different behavior was observed on the graphene surface, where the current stayed very low until $\approx 60-70{ }^{\circ} \mathrm{C}$ upon cooling, and then suddenly quickly increased by $\approx 10$ fold to reach a maximum value at room temperature. The mobility measured at room temperature was $\approx 4.2$ times higher on graphene than on silicon, reaching a value of $\mu \approx 5.0 \times 10^{-4} \mathrm{~cm}^{2} \mathrm{~V}^{-1} \mathrm{~s}^{-1}$.

Since charge transport in semiconducting polymer films strongly depends on the amount of crystallites and the crystalline orientation, the very different mechanisms of charge transport observed on $\mathrm{Si}$ and $\mathrm{G}$ suggest different orientations of crystallites, and also different kinetics of crystallization on each surface. The detailed process of crystallization of P3HT on both surfaces was investigated by in situ GIXD synchrotron measurements during cooling from $240{ }^{\circ} \mathrm{C}$, and is summarized in the ESI. $\dagger$ For clarity and ease of reading, we have focussed the following discussion on the interplay between charge transport and crystallite orientation.

At $240{ }^{\circ} \mathrm{C}$ both films were disordered as shown by the absence of any diffraction (Fig. S1b and c, ESI $\dagger$ ). Upon cooling, crystallites started to form primarily with in-plane $\pi$ stacking (edge-on configuration, Fig. 1a) on silicon, and with out-ofplane $\pi$ stacking (face-on, Fig. 1b) on graphene. The Hermans orientation parameter $S$, which indicates preferred orientation of crystallites in the films, was calculated from the diffracted intensity as a function of temperature (see Fig. 3a). The parameter $S$ is defined as: ${ }^{28,29}$

$$
\begin{gathered}
f_{\mathrm{p}}=\frac{\int_{0}^{\pi / 2} I(\chi) \cos ^{2} \chi \sin \chi \mathrm{d} \chi}{\int_{0}^{\pi / 2} I(\chi) \sin \chi \mathrm{d} \chi} \\
S=\frac{1}{2}\left(3 f_{\mathrm{p}}-1\right)
\end{gathered}
$$

where $\chi$ is the polar angle and $I(\chi)$ the diffracted intensity. A value of $S=1$ represents crystallites with edge-on configuration, while for $S=-0.5$ the crystallites are face-on. A value of $S=0$ denotes no particular orientation.

The calculation of the Hermans parameter (Fig. 3a) showed that crystallites were preferably oriented edge-on on silicon $(S \approx 0.2)$, and that a mixture of face-on and edge-on crystallites were found on graphene $(S \approx-0.03)$ at temperatures below $\approx 150{ }^{\circ} \mathrm{C}$.

Moreover, a non-negligible amount of randomly oriented crystallites, was also present as shown by the (100) ring at $q \approx 0.39 \AA^{-1}$ in ESI, $\dagger$ Fig. S1b and c, which explains the deviation of $S$ from either values of 1.0 or -0.5 . Polar plots quantitatively represent the diffracted intensity from the films at all angles (Fig. S2, ESI $\dagger$ ). A polar angle $\chi=2^{\circ}$ (next to $z$ axis) represents edge-on crystallites, whereas $\chi=90^{\circ}$ ( $x y$ plane) represents face-on crystallites. It can be seen that on graphene most random crystallites are found at polar angles $\chi \approx 45-88^{\circ}$ (see Fig. S1b, ESI $\dagger$ ), whereas on silicon the majority of randomly oriented crystallites are found at polar angles $\chi \approx 2-45^{\circ}$ (see Fig. S1c, ESI $\dagger$ ), extending from the $z$ axis where edge-on crystallites first formed. Calculation of the amount of
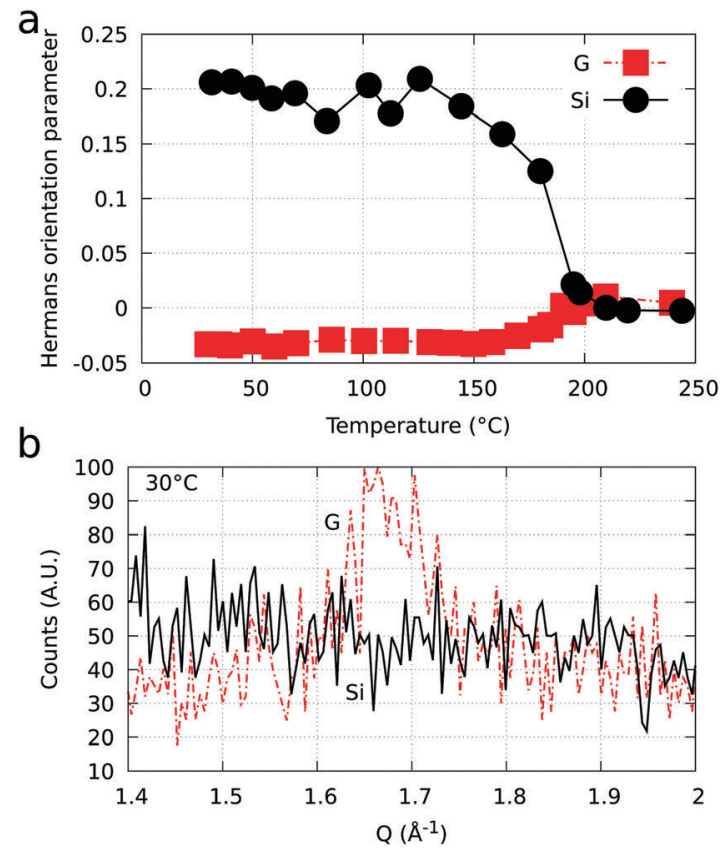

Fig. 3 Crystallite orientation on graphene and on silicon. (a) Hermans orientation parameter $S$ as a function of cooling temperature for the 100 peak on silicon (dot) and on graphene (square). (b) Specular Bragg diffraction peak for $\left(010_{z}\right)$ after cooling down to room temperature on both surfaces.

well-oriented face-on crystallites, defined here as the integrated intensity of the $(100)_{x y}$ peak divided by the sum of the integrated intensities of both the $(100)_{x y}$ and $(100)_{z}$ peaks at room temperature, gave $\approx 2 \%$ on silicon and $\approx 20$ times more on graphene. These results confirm the much larger amount of face-on crystallites found on graphene by specular X-ray diffraction (Fig. 3b), clearly observable by the well defined $(010)_{z}$ peak on graphene at room temperature while no peak was detected on silicon. This is indicative of the stronger $\pi-\pi$ interactions existing between graphene and polythiophene molecules which favor formation of face-on lamellae. It is therefore important to consider the different populations of crystallites on each substrate in order to better understand charge transport. Indeed, face-on lamellae favor vertical charge transport in the film whereas edge-on lamellae, which have vertically oriented insulating alkyl side chains, tend to hinder vertical charge transport. ${ }^{19}$

In order to distinguish between the different populations of crystallites in both films, we adopt the following nomenclature with respect to diffractions at $q \approx 0.39 \AA^{-1}$ (100 diffraction): (1) well-oriented edge-on and face-on lamellae are distinguished by diffraction spots at $\chi \approx 0-2^{\circ}$ from the $z$ axis, or the $x y$ plane, respectively; (2) a (100) diffraction with $\chi \approx 2-88^{\circ}$ represents non-oriented (random) crystallites. We moreover distinguish between non-oriented predominantly edge-on $\left(\chi \approx 2-45^{\circ}\right)$, and non-oriented predominantly face-on $\left(\chi \approx 45-88^{\circ}\right)$ crystallites. A discussion of how the crystallization of the P3HT films can help explain the increase in current and mobility on both surfaces, as a function of cooling temperature, is now presented.

The fast enhancement in charge transport which occurred on graphene can be linked to two noteworthy changes in 


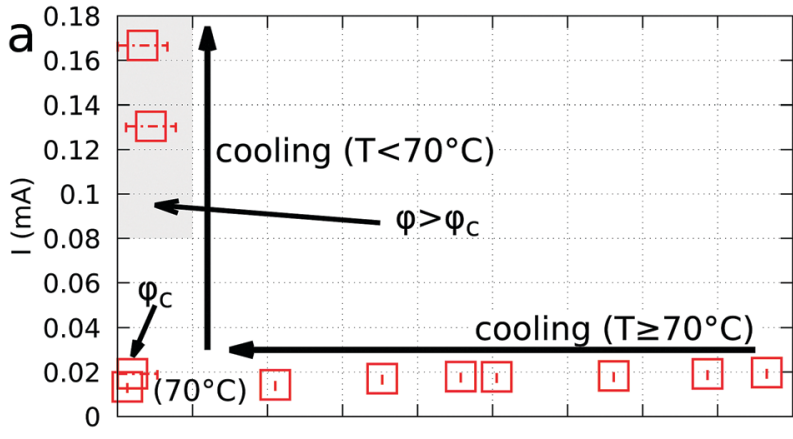

$\begin{array}{llllllllll}3.74 & 3.75 & 3.76 & 3.77 & 3.78 & 3.79 & 3.8 & 3.81 & 3.82 & 3.83\end{array}$ $\pi-\pi$ spacing $(\AA)$
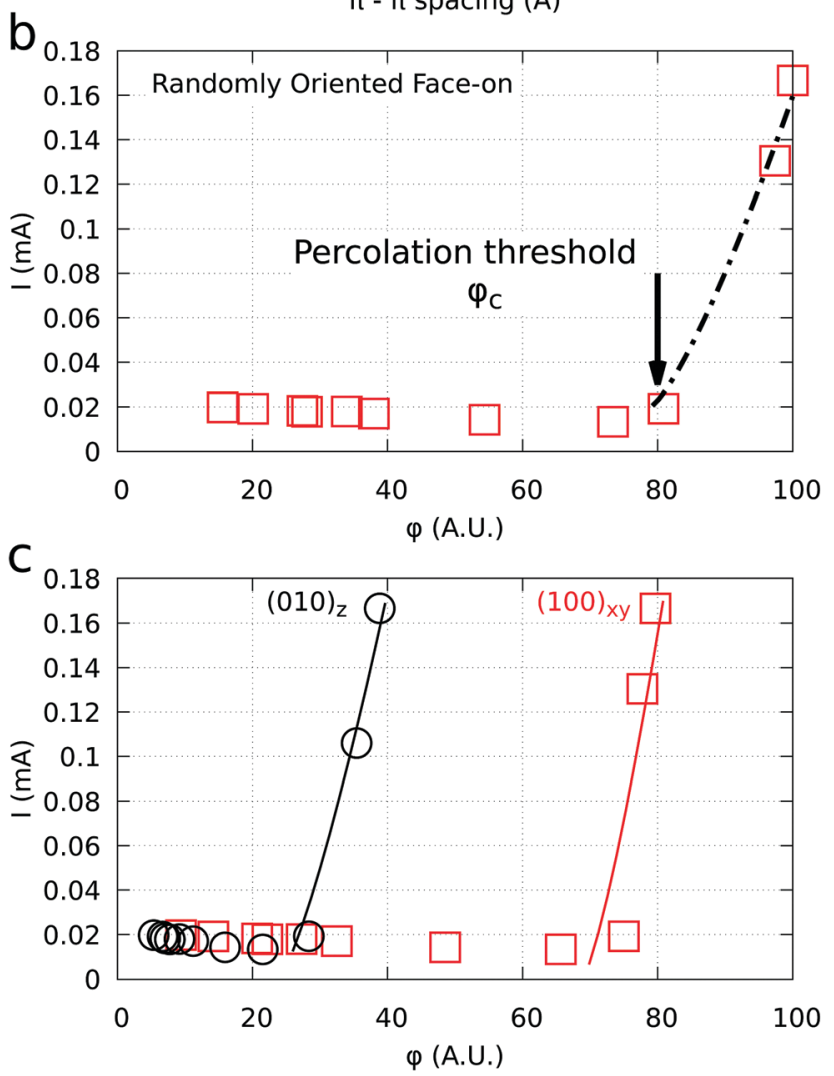

Fig. 4 Correlation between electrical current and grazing incidence X-ray diffraction data during cooling from the melt on graphene. (a) Evolution of the current vs. interplanar $\pi-\pi$ spacing for the face-on lamellae during cooling. The lowest $\pi-\pi$ spacing was measured just before percolation was reached $\left(\phi_{\mathrm{c}}\right)$, at which point the current increased by more than 10 fold upon further cooling $\left(T<70{ }^{\circ} \mathrm{C}\right)$. (b) Current vs. amount of nonoriented face-on crystallites (as in Fig. 3b) showing a correlation after the percolation threshold point at $70{ }^{\circ} \mathrm{C}$, where $d$ reached its lowest value. The films were probed with a synchrotron $\mathrm{X}$-ray beam at an incident angle $\alpha=0.13^{\circ}$. (c) Electrical current vs. integrated intensity of the $\left(100_{x y}\right)$ and $\left(010_{z}\right)$ peaks on graphene. The data was fitted with the percolation theory, where the solid lines are fits to eqn (4).

crystallinity. First, the $\pi-\pi$ distance of face-on crystallites reached a minimum and constant value of 3.74-3.75 $\AA$ (Fig. 4a). A shorter $\pi-\pi$ distance, has been previously shown to favor charge transport inside an aggregate for both small molecules, ${ }^{30,31}$ and for polymers. ${ }^{32,33}$ Second, the current increased only once a minimum amount of crystallites (face-on and non-oriented face-on) was reached in the film (Fig. 4), which occurred at $\approx 60-70{ }^{\circ} \mathrm{C}$ (see Fig. 2b). This sudden and fast increase in current is reminiscent of an electrical percolation where a continuous and unbroken path of interconnected entities is formed across the system at the percolation threshold $\left(\phi_{\mathrm{c}}\right)$. $^{34}$

In the case of a semiconducting polymer, the conductive entities are the crystallites and the tie-chains. The observation of percolation in polythiophene polymers has recently been reported by horizontal FET mobility measurements in films with a majority of edge-on aggregates. ${ }^{35}$ Here however, charges must be transported vertically along $\pi$ stacking oriented out-of-plane (face-on and nonoriented face-on lamellae linked through tie-chains, which are the majority crystallites in the film). Therefore the more crystalline the film, the easier it is for charges to find a path across multiple crystallites, and when a sufficient amount of properly oriented crystallites with out-of-plane $\pi$ stacking and connecting tie-chains is reached, percolation may occur and results in a fast increase in charge transport. Here we measured the amount of crystallites $\phi$ with their $\pi$ stacking oriented out-of-plane (face-on and nonoriented face-on) in the film on graphene, and plotted the current $v s$. $\phi$ using the electrical percolation equation: ${ }^{36}$

$$
I \propto\left(\phi-\phi_{\mathrm{c}}\right)^{t}
$$

where $I$ is the electrical current measured in the out-of-plane direction, $\phi$ represents the amount of crystallites in the film, $\phi_{\mathrm{c}}$ is the percolation threshold, and $t$ is the critical exponent. Note that usually eqn (4) is written with the conductivity $\sigma$ instead of current on the left hand side. The conductivity is defined as $\sigma=\frac{I}{U} \cdot \frac{t}{A}$, where $t$ is the film thickness and $A$ is the contact area between the top electrode and the film. Here, however the film thickness, electrode contact area and applied voltage $U$ were kept constant during the measurement. For simplicity, we therefore used the current $I$ instead of the conductivity in eqn (4) and in the remaining of the discussion. The amount of crystallites $\phi$ is directly measured from the integrated $\mathrm{X}$-ray diffracted intensity of the $100_{x y}$ or $010_{z}$ peaks in the case of face-on crystallites during crystallization. ${ }^{37}$

Current $v$ s. $\phi$ is displayed in Fig. $4 \mathrm{~b}$ and c, where the solid lines are fits of the data using eqn (4). The percolation threshold was determined using an implementation of the LevenbergMarquardt method by fitting the peaks of crystallites with outof-plane $\pi$ stacking (e.g. $010_{z}, 100_{x y}$ and randomly oriented face-on) with eqn (4). ${ }^{38}$ Values of $\phi_{c}$ are given in Table 1, along with the critical exponent $t$. In all cases, a critical exponent $t \approx 1.3$ was found, indicating the formation of a 2-dimensional network. ${ }^{39}$ As long as the minimum amount of crystallites needed for percolation had not been reached $\left(\phi<\phi_{\mathrm{c}}\right)$, the current remained low because an interconnected pathway has

Table 1 Values of the percolation threshold $\phi_{c}$ and of the critical exponent $t$ obtained by fitting using eqn (4) for the film on graphene

\begin{tabular}{lll}
\hline Diffraction peak & $\phi_{\mathrm{c}}$ (a.u.) & $t$ \\
\hline$\left(100_{x y}\right)$ & 68.85 & 1.3 \\
$\left(010_{z}\right)$ & 23.81 & 1.3 \\
Random orient. & 78.95 & 1.3
\end{tabular}


a

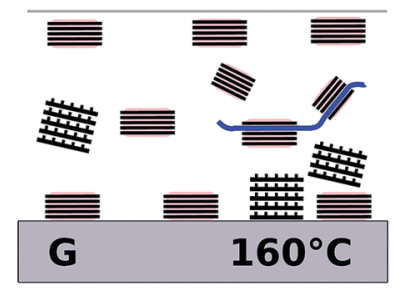

b

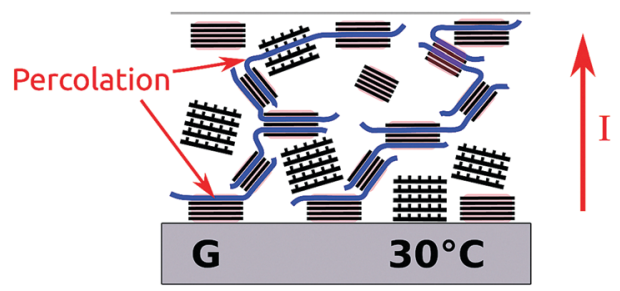

d

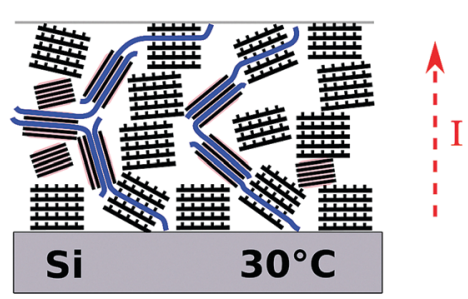

Edge-on

Face-on

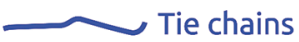

Fig. 5 Schematics of the formation of charge transport pathways in a P3HT film on graphene (a and b) and on silicon (c and d) at $160{ }^{\circ} \mathrm{C}$ and at $30{ }^{\circ} \mathrm{C}$. Non-oriented edge-on and face-on lamellae are represented with a tilt angle from the horizontal substrate. Tie-chains bridging crystallites are shown in blue. Charge transport is represented by a red dotted arrow (on Si) and a red solid arrow (on G) pointing upward.

not been created yet throughout the film's thickness. Above $\phi_{\mathrm{c}}$, a fast increase in current was measured indicating the formation of a percolating network of conducting crystallites on graphene (see Fig. $4 \mathrm{~b}$ and c).

We propose a schematic model which illustrates the differences in crystallinity and charge transport of $\mathrm{P} 3 \mathrm{HT}$ on graphene and silicon (Fig. 5a and b). At higher temperatures (e.g. $\approx 160{ }^{\circ} \mathrm{C}$ ), the amount of crystallites with out-of-plane $\pi$ stacking was too low on graphene to enable charge transport through the thickness of the film (Fig. 5a). As the film cools down, more crystallites are formed and below $\approx 60{ }^{\circ} \mathrm{C}$ percolation occurred with the formation of continuous pathways throughout the film's thickness, which enable charge transport across multiple crystallites bridged by tie-chains (Fig. 5b). The reduction in $\pi-\pi$ distance of face-on lamellae (Fig. 4a), reaching a low value at the same temperature, is also likely to help faster vertical charge transport. It is worth noticing that the overall amount of crystallites on graphene was still relatively low, and only $\approx 36 \%$ of that measured in film grown on silicon (Fig. S3, ESI $\dagger$ ). On silicon indeed, the amount of crystallites was already much larger at elevated temperatures $\left(\right.$ e.g. $\approx 160{ }^{\circ} \mathrm{C}$ ), enabling weak charge transport through interconnected randomly oriented crystallites linked by tie-chains (Fig. 5c). The Hermans orientation parameter $S \approx 0.15-0.2$ indeed suggest a mix of edge-on and randomly orientated crystallites on silicon (Fig. 3). As more and more crystallites formed during further cooling, a steady current increase was measured as more pathways for charge transport were created, reaching a maximum current value around 80$100{ }^{\circ} \mathrm{C}$. The current however remained much lower on silicon because of the less efficient transport of charges through edge-on lamellae compared to face-on lamellae. For lower temperatures, a slight decrease in current was measured on silicon (Fig. 2b) which is explained by the fact that charge transport is thermally activated in disordered systems such as $\mathrm{P} 3 \mathrm{HT} .{ }^{40}$ The mobility has been shown to follow an Arrhenius-like temperature dependence as previously observed, ${ }^{41}$ and therefore both mobility and current are expected to decrease, in a film whose crystalline order does not change significantly, when cooling down in the temperature range investigated.

\section{Conclusions}

In conclusion, we have performed a parallel series of experiments to determine in situ the crystallinity and the charge transport on P3HT films on both silicon and graphene substrates as a function of the cooling temperature from the melt. Our results show that single layer graphene can be used at the interface with a semiconducting polythiophene polymer film in order to strongly enhance charge transport by a percolation induced mechanism, which was not found on the less interacting silicon interface. Charges are transported principally through face-on and non-oriented face-on crystallites on graphene, whereas non-oriented edge-on crystallites (and tie-chains bridging them) are believed to be the main mechanisms of charge transport on silicon. These results provide a better understanding of how semiconducting polymer films crystallize on graphene, and show the importance of the chemistry of the interface and of its interaction with the polymer crystallites, which influence not only the crystallization but also the formation of electrical pathways in the $\mathrm{P} 3 \mathrm{HT}$ film. At percolation, a fast increase in current was measured in $\mathrm{P} 3 \mathrm{HT}$ deposited on graphene, and lead to a mobility $\approx 4.2$ times higher compared to silicon. 


\section{Conflicts of interest}

There are no conflicts to declare.

\section{Acknowledgements}

Portions of this research were carried out at the Stanford Synchrotron Radiation Lightsource, a Directorate of SLAC National Accelerator Laboratory and an Office of Science User Facility operated for the U.S. Department of Energy Office of Science by Stanford University. We thank Dr V. Skrypnychuk for help with the electrical measurements. The authors thank the Baltic Foundation, the Kempe Foundation and a STINT grant for financial support. D. R. B. also thanks a Young Researcher Career Award from Umeå University for support of this work.

\section{References}

1 K. S. Novoselov, A. K. Geim, S. V. Morozov, D. Jiang, Y. Zhang, S. V. Dubonos, I. V. Grigorieva and A. A. Firsov, Science, 2004, 306, 666-669.

2 B. Obradovic, R. Kotlyar, F. Heinz, P. Matagne, T. Rakshit, M. D. Giles, M. A. Stettler and D. E. Nikonov, Appl. Phys. Lett., 2006, 88, 142102.

3 Z. Liu, Q. Liu, Y. Huang, Y. Ma, S. Yin, X. Zhang, W. Sun and Y. Chen, Adv. Mater., 2008, 20, 3924-3930.

4 L. Wang, I. Meric, P. Y. Huang, Q. Gao, Y. Gao, H. Tran, T. Taniguchi, K. Watanabe, L. M. Campos, D. A. Muller, J. Guo, P. Kim, J. Hone, K. L. Shepard and C. R. Dean, Science, 2013, 342, 614-617.

5 L. Zhang, Y. Li, J. Shi, G. Shi and S. Cao, Mater. Chem. Phys., 2013, 142, 626-632.

6 Y.-C. Lai, Y.-X. Wang, Y.-C. Huang, T.-Y. Lin, Y.-P. Hsieh, Y.-J. Yang and Y.-F. Chen, Adv. Funct. Mater., 2013, 24, 1430-1438.

7 Y. Wu, X. Zhang, J. Jie, C. Xie, X. Zhang, B. Sun, Y. Wang and P. Gao, J. Phys. Chem. C, 2013, 117, 11968-11976.

8 J. Huang, D. R. Hines, B. J. Jung, M. S. Bronsgeest, A. Tunnell, V. Ballarotto, H. E. Katz, M. S. Fuhrer, E. D. Williams and J. Cumings, Org. Electron., 2011, 12, 1471-1476.

9 V. Skrypnychuk, N. Boulanger, V. Yu, M. Hilke, M. F. Toney and D. R. Barbero, J. Mater. Chem. C, 2016, 4, 4143-4149.

10 G. Wang, J. Swensen, D. Moses and A. J. Heeger, J. Appl. Phys., 2003, 93, 6137-6141.

11 H. Yang, T. Shin, L. Yang, K. Cho, C. Ryu and Z. Bao, Adv. Funct. Mater., 2005, 15, 671-676.

12 M. Surin, P. Leclère, R. Lazzaroni, J. D. Yuen, G. Wang, D. Moses, A. J. Heeger, S. Cho and K. Lee, J. Appl. Phys., 2006, 100, 033712.

13 D. Gargi, R. J. Kline, D. M. DeLongchamp, D. A. Fischer, M. F. Toney and B. T. O'Connor, J. Phys. Chem. C, 2013, 117, 17421-17428.

14 L. H. Jimison, S. Himmelberger, D. T. Duong, J. Rivnay, M. F. Toney and A. Salleo, J. Polym. Sci., Part B: Polym. Phys., 2013, 51, 611-620.

15 W. Porzio, G. Scavia, L. Barba, G. Arrighetti and S. Milita, Eur. Polym. J., 2011, 47, 273-283.
16 D. H. Kim, H. S. Lee, H.-J. Shin, Y.-S. Bae, K.-H. Lee, S.-W. Kim, D. Choi and J.-Y. Choi, Soft Matter, 2013, 9, 5355-5360.

17 S. B. Jo, H. H. Kim, H. Lee, B. Kang, S. Lee, M. Sim, M. Kim, W. H. Lee and K. Cho, ACS Nano, 2015, 9, 8206-8219.

18 D. Yu, Y. Yang, M. Durstock, J.-B. Baek and L. Dai, ACS Nano, 2010, 4, 5633-5640.

19 V. Skrypnychuk, N. Boulanger, V. Yu, M. Hilke, S. C. B. Mannsfeld, M. F. Toney and D. R. Barbero, Adv. Funct. Mater., 2015, 25, 664-670.

20 D. R. Cooper, B. D’Anjou, N. Ghattamaneni, B. Harack, M. Hilke, A. Horth, N. Majlis, M. Massicotte, L. Vandsburger, E. Whiteway and V. Yu, ISRN Condens. Matter Phys., 2012, 2012, 501686.

21 V. Yu, E. Whiteway, J. Maassen and M. Hilke, Phys. Rev. B: Condens. Matter Mater. Phys., 2011, 84, 205407.

22 J. Rivnay, R. Noriega, R. J. Kline, A. Salleo and M. F. Toney, Phys. Rev. B: Condens. Matter Mater. Phys., 2011, 84, 045203.

23 V. Skrypnychuk, G.-J. A. H. Wetzelaer, P. I. Gordiichuk, S. C. B. Mannsfeld, A. Herrmann, M. F. Toney and D. R. Barbero, Adv. Mater., 2016, 28, 2359-2366.

24 B. de Boer, A. Hadipour, M. M. Mandoc, T. van Woudenbergh and P. W. M. Blom, Adv. Mater., 2005, 17, 621-625.

25 M. Osaka, H. Benten, L.-T. Lee, H. Ohkita and S. Ito, Polymer, 2013, 54, 3443-3447.

26 M. C. Scharber, D. Mühlbacher, M. Koppe, P. Denk, C. Waldauf, A. J. Heeger and C. J. Brabec, Adv. Mater., 2006, 18, 789-794.

27 N. F. Mott and R. W. Gurney, Electronic Processes in Ionic Crystals, Dover Publications, New York, United States, 1964.

28 L. A. Perez, P. Zalar, L. Ying, K. Schmidt, M. F. Toney, T.-Q. Nguyen, G. C. Bazan and E. J. Kramer, Macromolecules, 2014, 47, 1403-1410.

29 K.-Y. Wu, C.-T. Hsieh, L.-H. Wang, C.-H. Hsu, S.-T. Chang, S.-T. Lan, Y.-F. Huang, Y.-M. Chen and C.-L. Wang, Cryst. Growth Des., 2016, 16, 6160-6166.

30 G. Giri, E. Verploegen, S. C. B. Mannsfeld, S. Atahan-Evrenk, D. H. Kim, S. Y. Lee, H. A. Becerril, A. Aspuru-Guzik, M. F. Toney and Z. Bao, Nature, 2011, 480, 504-508.

31 G. Giri, S. Park, M. Vosgueritchian, M. M. Shulaker and Z. Bao, Adv. Mater., 2014, 26, 487-493.

32 I. Kang, H.-J. Yun, D. S. Chung, S.-K. Kwon and Y.-H. Kim, J. Am. Chem. Soc., 2013, 135, 14896-14899.

33 B. Sun, W. Hong, Z. Yan, H. Aziz and Y. Li, Adv. Mater., 2014, 26, 2636-2642.

34 G. Pike and C. Seager, Phys. Rev. B: Solid State, 1974, 10, 1421.

35 D. T. Duong, V. Ho, Z. Shang, S. Mollinger, S. C. Mannsfeld, J. Dacuña, M. F. Toney, R. Segalman and A. Salleo, $A d v$. Funct. Mater., 2014, 24, 4515-4521.

36 J. W. Essam, Rep. Prog. Phys., 1980, 43, 833-912.

37 X. Shen, W. Hu and T. P. Russell, Macromolecules, 2016, 49, 4501-4509.

38 D. W. Marquardt, J. Soc. Ind. Appl. Math., 1963, 11, 431-441. 39 D. Stauffer and A. Aharony, Introduction to Percolation Theory, Taylor \& Francis, 1992.

40 V. Coropceanu, J. Cornil, D. A. da Silva Filho, Y. Olivier, R. Silbey and J.-L. Brédas, Chem. Rev., 2007, 107, 926-952.

41 C. Goh, R. J. Kline, M. D. McGehee, E. N. Kadnikova and J. M. J. Fréchet, Appl. Phys. Lett., 2005, 86, 122110. 